\title{
Collective Instabilities in the Tevatron Complex
}

\author{
Valeri Lebedev and Alexey Burov
}

FNAL, P.O. Box 500, Batavia, IL 60510, USA

\begin{abstract}
Although the luminosity growth for Tevatron Run II was slower than expected, steady growth of luminosity has been demonstrated during the last three years with the peak luminosity of $1.02 \times 10^{32} \mathrm{~cm}^{-2} \mathrm{~s}^{-1}$ achieved in July 2004 . Suppression of instabilities has been a valuable contributor to the luminosity growth. This report discusses the transverse instabilities in the Tevatron and Recycler.
\end{abstract}

\section{INTRODUCTION}

The luminosity growth demonstrated during Tevatron Run II has been achieved through large number of improvements for each of circular machines, as well as for the linac and multiple transfer lines. Although each improvement separately did not produce significant luminosity growth, altogether they contributed more than tenfold luminosity growth during Run II started in the middle of 2001. Five of six rings (except Debuncher) have sufficiently large beam intensity and related problems with beam stability. The instabilities of almost every type are present there: single and multi-bunch, transverse and longitudinal, due to electromagnetic interaction with vacuum chamber and due to interaction with ions stored in the beam. In this report, we discuss only transverse instabilities in Tevatron and Recycler.

\section{HEAD-TAIL INSTABILITY IN TEVATRON AND ITS SUPPRESSION}

Transverse head-tail instability has been a longstanding problem for the Tevatron. It forced to keep high chromaticities, yielding reduced beam lifetime. A detailed experimental study was presented in Ref. [1]. The instability was diagnosed as the single-bunch instability, although some couple-bunch contributions were not being excluded. Experimentally, it was not clear whether it was a weak or strong head-tail case. Calculations showed that it was at the margin. The problem has been alleviated after two step reduction of Tevatron impedance in 2002 and 2003.

\subsection{Source of the Impedance}

Instability growth rate was measured at beam injection. To make it more pronounced, the chromaticities were set to small negative values. Monte-Carlo single-bunch code with resistive wake and Gaussian beam distribution was used to compute the impedance from the measurements. It yielded the transverse resistive impedance $Z \geq 4 M \Omega / m$ at 100 MHz. It was expected that the vacuum chamber resistivity should make the main contribution. The measured value was about 4 times higher. Consequent analysis has shown that this difference was related to the contribution of laminated Lambertson magnets. Their impedance can be calculated by integrating the resistance over the low frequency current passing along the laminations, leading to [2]:

$$
Z=(1-i) \frac{g Z_{0} L}{2 \pi a^{3}} \frac{c}{\sqrt{2 \pi \sigma \omega}} \frac{2 a \sqrt{\mu}}{d}
$$

Here $a$ is the chamber radius, or a half-gap for a flat chamber, $L$ is the magnet length, $d$ is the lamination thickness, $\mu$ is the magnetic permeability, $g$ is the geometrical Yokoya factor ( $g=1$ for round chambers), and $Z_{0}=377 \Omega$.

When a reason of the instability was understood, one Lambertson magnet (unused) was removed, and another (injection magnet) was shielded. Presently, the transverse impedance is dominated by wall resistivity of the main vacuum chamber (stainless steel), so the total impedance was reduced by a factor of 3-4. 


\subsection{Single and Coupled Bunch Contributions}

Within the air-bag (hollow-bunch) approximation, beam transverse modes are characterized by singlebunch, or head-tail, number $l$, and a coupled-bunch number $m$. The head-tail motion is driven by highfrequency impedance, at $\omega \geq c / \sigma_{z}$, or $f \geq 50 \mathrm{MHz}$ for the Tevatron, while the coupled-bunch motion is related to much lower frequencies, approximately between the revolution frequency and bunch-to-bunch frequency, $\omega_{0} \leq \omega \leq N \omega_{0}$. A coherent tune shift of a given mode linearly depends on the impedance. Because the single- and coupled-bunch contributions are driven by the well separated frequency ranges of the impedance, the total growth rate is a sum of single and multi bunch rates: $\Lambda_{l m}=\Lambda_{l}^{s}+\Lambda_{l m}^{c}$. Following [3],

$$
\begin{aligned}
& \Lambda_{l}^{s}=-\frac{N_{b} r_{0}}{2 \pi Z_{0} \gamma v_{\beta}} \int_{-\infty}^{\infty} d \omega \operatorname{Re} Z(\omega) J_{l}^{2}\left(\omega z_{0} / c-\chi\right), \\
& \Lambda_{l m}^{c}=-\frac{N N_{b} r_{0} \omega_{0}}{2 \pi Z_{0} \gamma v_{\beta}} J_{l}^{2}(\chi) \sum_{p=-\infty}^{\infty} \operatorname{Re} Z\left(\omega_{0}\left(v_{\beta}+p N+m\right)\right) .
\end{aligned}
$$

where $\chi$ is the head-tail phase, $z_{0}$ is the longitudinal amplitude, and all other notations are conventional. The two contributions are comparable for the Tevatron. Their behavior with the chromaticity is shown in Figure (1), assuming the resistive wall impedance. At low chromaticity end, single-bunch contributions clearly dominate.

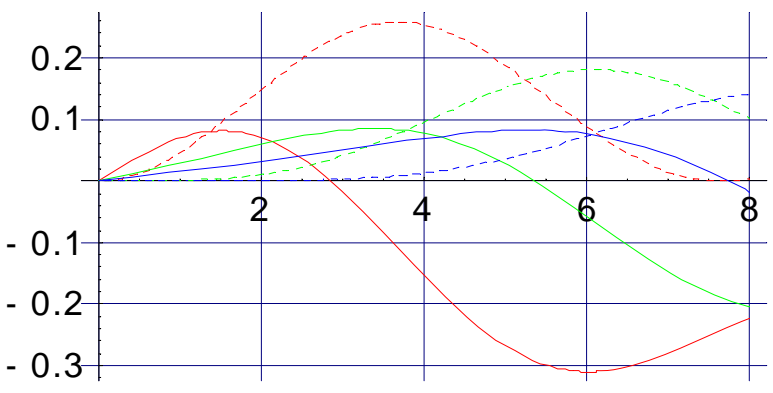

Figure 1. Single-bunch (solid) and the most unstable coupled-bunch (dashed) growth rate contributions to $l=1$ (red), $l=2$ (green) and $l=3$ (blue) head-tail modes, shown versus the chromaticity. Units for the rates are arbitrary.

\subsection{Damper Optimization}

A transverse bunch-by-bunch damper was built before the problem with Lambertson magnets was resolved. It is still used during beam injection and acceleration. Its scheme is sketched in Fig. 2. Use of this damper allowed decreasing the chromaticity from

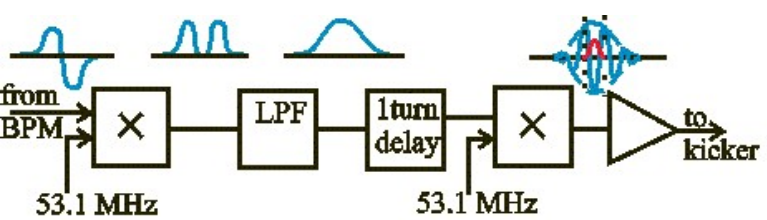

Figure 2. Transverse damper layout; signal (blue) is mixed with the $53 \mathrm{MHz}$ RF frequency, integrated by the low-pass filter, delayed 1 turn, mixed again with $53 \mathrm{MHz}$, and applied to the kicker.

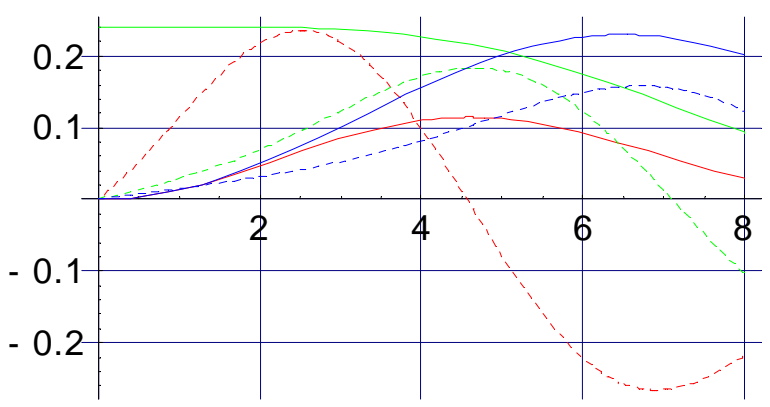

Figure 3. Instability rates (dashed) and damping rates (solid) versus chromaticity for the same modes as Fig.1. The mode $l=1$ gets unstable at the chromaticity $\sim 4$. The mixing phase shift $\theta=0$.

$\sim 6$ to $\sim 4$ units (un-normalized), but at lower chromaticity the beam was still unstable.

For air-bag distribution, the damping rates are:

$$
\Gamma_{l}=g\left|J_{l}(\chi+q) e^{i \theta}+J_{l}(\chi-q) e^{-i \theta}\right|^{2},
$$

where $g$ is a constant related to the amplification coefficient, $q=\omega_{R F} z_{0} / c$. The parameter $\theta$ is the phase shift of mixing frequency determined to be $\propto \sin \left(q z / z_{0}+\theta\right)$ at the pickup, and $\propto \cos \left(q z / z_{0}+\theta\right)$ at the kicker. Such phase correlation guarantees positive sign of the introduced damping rate for all the modes. Choice of the phase shift $\theta$ is a matter of optimization. Until now the damper has been operated for only $\theta=0$ and as one can see from Eq. (3) it damps both the dipole and head-tail modes. The second was not anticipated at the design stage and came as a pleasant surprise. A comparative behavior of the instability rate $\Lambda_{l m}$ and the damping rate $\Gamma_{l}$ for the first 3 head-tail modes is shown in Fig. 3 . Note that at low chromaticity, $\chi \leq 1$, the instability dominates over damping: $\Lambda_{l} \propto \chi$, while $\Gamma_{l} \propto \chi^{2}$ for odd modes and $\theta=0$; and the main stopper is $l=1$ mode. Thus, for $\theta=0$ the threshold chromaticity can be reduced but the instability cannot be suppressed at low chromaticities. Both statements agree with the observations. The opposite choice, $\theta=90^{\circ}$, would be 
about as bad, killing damping of dipole mode and making $l=2$ mode the low chromaticity stopper. The optimum should be expected somewhere in the middle. The same comparison is shown in Fig. 4 for $\theta=30^{\circ}$. Such choice damps dipole and all head tail modes and can drastically improve beam stability.

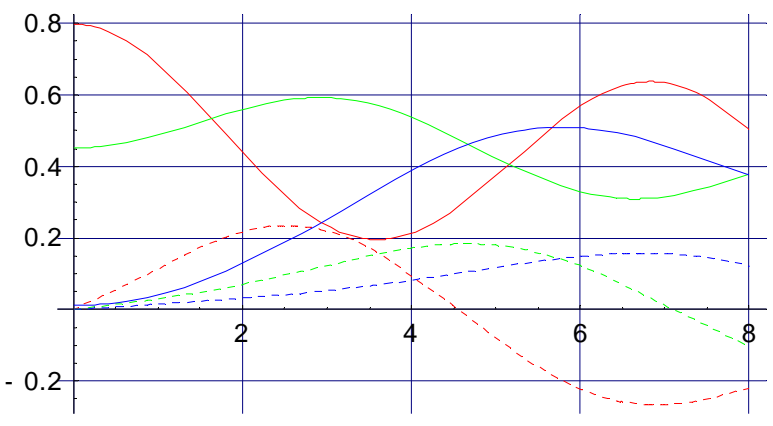

Figure 4. The same as Fig. 3, except $\theta=30^{\circ}$.

\section{RESISTIVE WALL INSTABILITY IN RECYCLER}

Recycler is $8 \mathrm{GeV}$ storage ring which is presently under commissioning in Fermilab. It was built as additional antiproton accumulator to boost the antiproton production and their total intensity. Both the stochastic and electron cooling are planned to be used. Fully operational Recycler should increase both the antiproton production rate and the maximum beam intensity by about 4 times so that $6 \cdot 10^{12}$ antiprotons would be accumulated after 15 hours storage. The ring circumference is $3328 \mathrm{~m}$, and the betatron tunes are $v_{x} / v_{y}=25.58 / 24.42$. To prevent the accumulation of ions produced by the beam from the residual gas and to decrease the beam longitudinal emittance the Recycler beam is stored in one long ( 600-1500 m) bunch kept by the barrier bucket RF. It creates the potential well with rigid walls at bunch ends making the rectangular longitudinal density distribution.

\subsection{Experimental measurements}

Recent measurements, performed with beam intensity of about $10^{12}$, exhibited that if the machine chromaticity is close to zero and the beam is sufficiently cold there appears a transverse instability. This instability causes particle loss but is followed by a comparatively little emittance growth; so that after beam stabilization the rms beam emittance is more than an order of magnitude smaller than the vacuum chamber acceptance. This and a few other peculiarities forced us to carry out detailed studies of the instability.
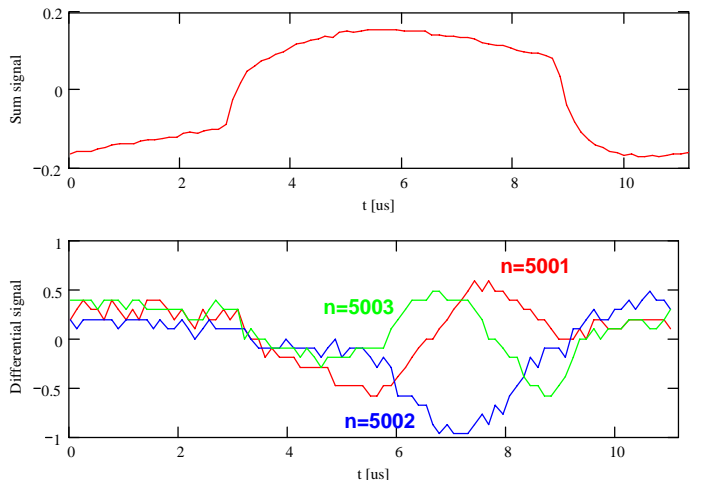

Figure 5. Signals from longitudinal and transverse pickups for three consecutive turns; number of particles $-10^{12}$, time of one full revolution is presented.

A fast digital oscilloscope connected to the sum and differential outputs of vertical pickups and triggered by the beam instability recorded a continuous signal for almost 90,000 turns with 128 ns sampling time. Figure 5 presents data for three consecutive turns. One can clearly see the betatron motion wave running along the bunch. To separate the betatron and head-tail motions the betatron phases and amplitudes along the bunch were computed from each three consecutive turns. The results are shown in Figure 6. The amplitude of the motion grows from the head to the tail, reaches its maximum at $\sim 2 / 3$ of the bunch length, and then decreases. The shape of the amplitude waveform and the betatron phase advance along the bunch are not changed in the course of instability development pointing out that only one unstable mode dominates the instability. Figure 7 presents changes in the amplitude and phase for fixed point in the bunch. The accuracy of phase calculations is so good that the phase changes due to tune modulation at power line frequencies are clearly seen. Fitting yields amplitudes of tune modulation to be 4.6.1 $10^{-4}$ and $3.2 \cdot 10^{-4}$ for the first and third power line harmonics, correspondingly.

\subsection{Theoretical model}

Because of large bunch length and small momentum spread the synchrotron motion is much slower than the instability. For Recycler the synchrotron motion period is $\sim 1 \mathrm{~s}$, while the instability growth time less than $20 \mathrm{~ms}$. In this case one can neglect the longitudinal particle displacement in the course of instability development. The instability would not develop in the absence of the tail-to-head feedback. It is created by the wake-fields delayed by 
one turn. The Recycler transverse impedance is dominated by the resistive wall impedance with slowly decaying wake, $\propto \sqrt{s}$. Neglecting the particle longitudinal motion and the spread of betatron tunes one obtains the motion equation in the beam frame:

$$
\frac{d^{2} x(s, t)}{d t^{2}}+\left(\omega_{b}^{2}-\frac{e^{2} N_{b}}{M \gamma} \tilde{D}(s)\right) x(s, t)=\frac{e^{2} N_{b}}{M \gamma} \tilde{W}(s) .
$$

Here $\widetilde{W}(s)$ and $D(s)$ represent the integral effect of the normal and detuning (quadrupole) wakes:
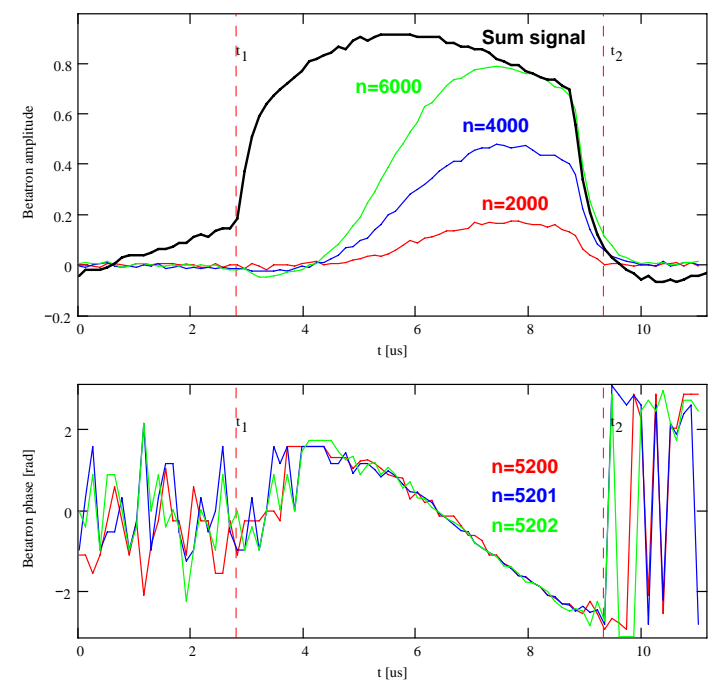

Figure 6. Amplitudes and betatron phases (referenced to the bunch head) along the bunch for turns 2000, 4000 and 6000.
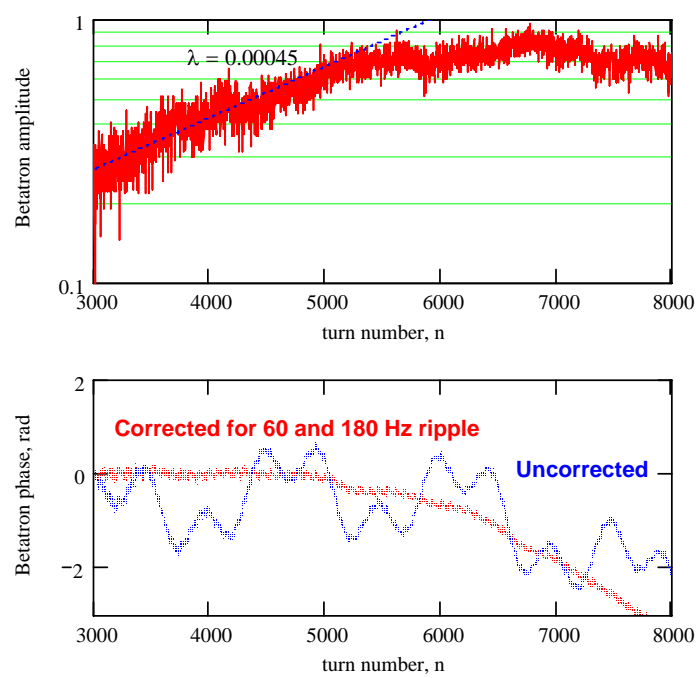

Figure 7. Dependence of betatron amplitude and phase on the turn number.

$$
\begin{aligned}
\tilde{D}(s) & =\int_{s}^{L} D\left(s^{\prime}-s\right) \rho\left(s^{\prime}\right) d s^{\prime}+\sum_{n=1}^{\infty} \int_{0}^{L} D\left(s^{\prime}+n C-s\right) \rho\left(s^{\prime}\right) d s^{\prime}, \\
\tilde{W}(s) & =\int_{s}^{L} W\left(s^{\prime}-s\right) \rho\left(s^{\prime}\right) x\left(s^{\prime}, t-\frac{s^{\prime}-s}{\mathrm{v}_{0}}\right) d s^{\prime}+ \\
& \sum_{n=1}^{\infty} \int_{0}^{L} W\left(s^{\prime}+n C-s\right) \rho\left(s^{\prime}\right) x\left(s^{\prime}, t-\frac{s^{\prime}+n C-s}{\mathrm{v}_{0}}\right) d s^{\prime} .
\end{aligned}
$$

Recycler has an elliptical vacuum chamber with large aspect ratio, which impedance is close to the impedance of flat chamber. For frequencies of interest, the impedance is dominated by wall resistivity yielding horizontal and vertical wakes:

$$
\begin{aligned}
& W_{\mathrm{v}}(s)=2 W_{h}(s)=\frac{\pi C}{6 a^{3}} \sqrt{\frac{\mathrm{v}_{0}}{\sigma s}}, \\
& D_{\mathrm{v}}(s)=-D_{h}(s)=\frac{\pi C}{12 a^{3}} \sqrt{\frac{\mathrm{v}_{0}}{\sigma s}} .
\end{aligned}
$$

The solution of Eq. (4) was carried out numerically for 600 macro-particles and the vertical resistive wall impedance $^{1}$ of Eq. (5). The results are presented in Figure 8. There is good agreement with analytical model for continuous beam $(B=1)$ and small mode numbers $(\mathrm{n} \leq 10)$. Divergence for large mode numbers (two bottom curves) is related to insufficient number of particles per oscillation length. For fixed number of particles, bunching of the beam increases the growth rate. For small bunching factor, $B$, the rate is $\propto 1 / B^{1 / 4}$, in agreement with a two-particle model. Figure 9 presents structure of the first three unstable modes. The structure of the lowest mode is close to the measurements presented in Figure 6. The measured and computed growth rates for this most unstable mode coincide within $10-20 \%$. That verifies that at low frequencies the impedance is dominated by the

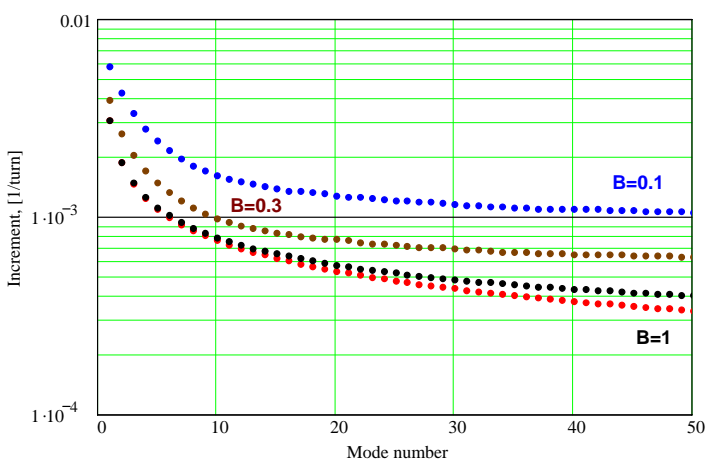

Figure 8. Instability growth rates of unstable modes for different bunching factors, $B ; N_{b}=6 \cdot 10^{12}$.

\footnotetext{
${ }^{1}$ The vertical impedance is larger and, consequently, the vertical degree of freedom is more unstable.
} 

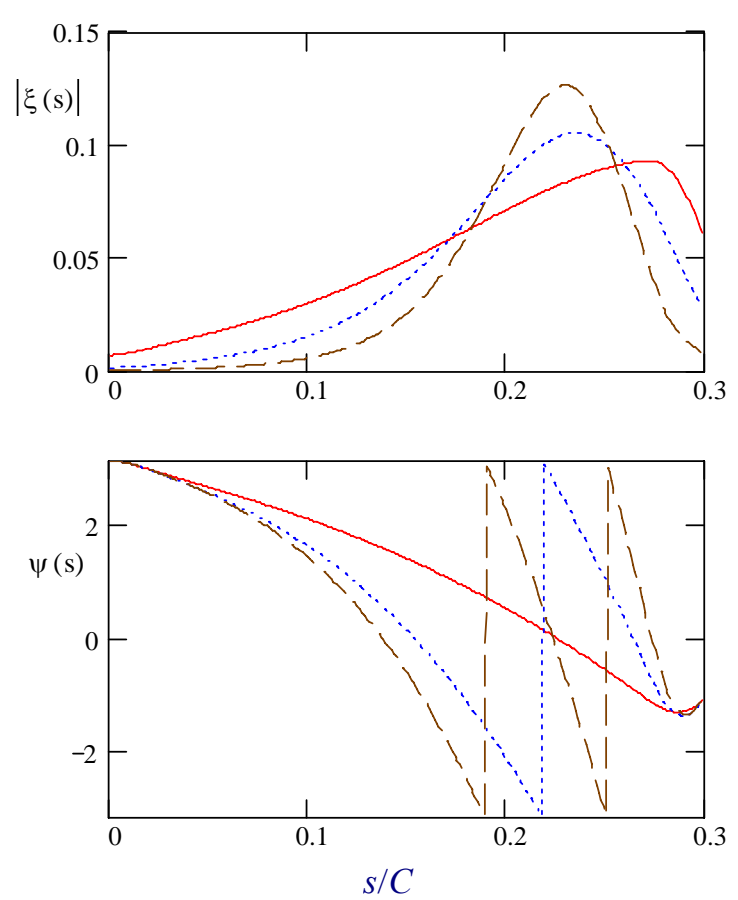

Figure 9. Amplitudes, $|\xi(s)|$, and phases, $\psi(s)$, along the bunch for first 3 unstable modes.
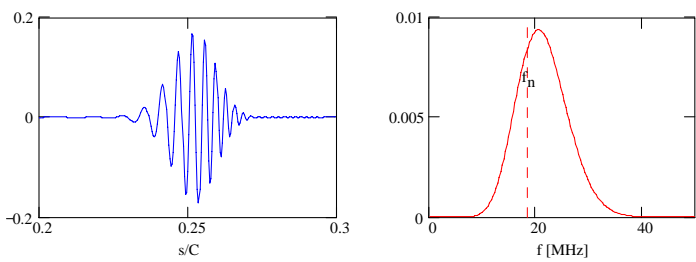

Figure 10. Structure, $\operatorname{Re}(\xi(s))$, and spectrum of mode $m=$ $101, \mathrm{~B}=0.3$.

wall resistivity of stainless steel vacuum chamber. Note that the detuning wake makes the betatron tunes dependent on particle position along the bunch. That results in achieving the peak in the amplitude at about $2 / 3$ of bunch length. For the round vacuum chamber the detuning wake is zero and simulations yield that the maximum amplitude is achieved at the bunch tail. Every next mode has the head to tail betatron phase advance increased by $2 \pi$ and the more peaked amplitude. Figure 10 shows the mode 101 and its Fourier spectrum. One can see that the frequency of oscillations grows from head to tail bringing additional increase of the spectrum width. For $B<<1$ and $n>>1$ the maximum of spectral density is achieved at frequency

$$
f_{n} \approx \frac{2.5 n}{B} f_{0}
$$

For a given mode number, comparing to the continuous beam the bunched beam has an increased instability growth rate and an increased oscillation frequency (measured at pickup). Both of them do not benefit the instability damping.

\subsection{Requirements for instability damping}

The betatron tune spread

$$
\Delta v_{n} \approx\left(\eta n-v^{\prime}\right) \frac{\Delta p}{p}
$$

gives rise to beam stabilization, Landau damping. In the absence of the space charge, all modes with $n \geq 3$ would be stabilized. However, the required phase space of the antiproton beam is so small that the space charge (incoherent) tune shift is expected to be quite large $\Delta v_{s c} \approx 0.03-0.1$. It will suppress the Landau damping up to very high frequencies

$$
\Delta v_{n} \approx \Delta v_{s c} / F_{s c} \quad, \quad F_{s c} \approx 3-6 .
$$

As a result, the required frequency band of the instability damper goes to well above $100 \mathrm{MHz}$.

\section{DISCUSSION}

Presently, instabilities do not produce severe limitations on the collider luminosity. Transverse instabilities in Tevatron and Recycler are well understood. We plan further reduction of the Tevatron chromaticity. Introducing cubic (octupole) nonlinearity is the main direction. Further improvements of head-tail damper may be required if operational difficulties with octupoles will be encountered. To suppress Recycler instability, the two band transverse damper: $10 \mathrm{kHz}-10 \mathrm{MHz}$ and $10-300 \mathrm{MHz}$ is planned to be built.

\section{ACKNOWLEDGMENTS}

The authors thank V. Shiltsev, P. Ivanov, S. Nagaitsev, J. Crisp, V. Scarpine, E. Lorman for discussions and sharing experimental data.

\section{REFERENCES}

1. P. M. Ivanov et al., Head-Tail instability at Tevatron, PAC 2003, p. 3062.

2. A. Burov and V. Danilov, private communication, 2002.

3. A. W. Chao, Physics of Collective Beam Instabilities, J. Wiley \& Sons, Inc, 1993. 